\title{
Modeling of the stamping process of box-type forging
}

\author{
VagidKadymov $^{1}$, EvgenySosenushkin ${ }^{2 *}$, and Elena Yanovskaya ${ }^{2}$ \\ ${ }^{1}$ Moscow State University of Humanities and Economics, Russia \\ ${ }^{2}$ Moscow State Technological University "STANKIN", Russia
}

\begin{abstract}
A new mathematical model for manufacturing rectangular of box-type forgings is proposed. Along the outer contour of the forging there are walls with thickness comparable to the thickness of the bottom part of the forging. To calculate the power parameters of the technological process under study, the authors use the theory of flow in a relatively thin plastic layer as described by A. A. Ilyushin. A thickness-averaged twodimensional mathematical model of the plastic layer spreading process is described by nonlinear partial differential equations of the first order with respect to the contact pressure and the flow velocity components. For a metal that is in a flat deformed state, the condition of complete plasticity is assumed, under which the tangent stresses in the layer are small in comparison with normal stresses and they can be ignored, and two normal stresses in the cross section can be considered equal in the first approximation. The third normal stress is equal to the contact pressure of the layer on the tool. In this case, it is sufficient to set a single boundary condition on the border of the regions.
\end{abstract}

\section{Introduction}

We consider box forging of rectangular shape, the walls of which have rounded corners. When stamping, the metal is deformed in accordance with the specified movement of the working parts of the stamp, while the latter are assumed to be rigid non-deformable. The thickness of the bottom part of the forging $h(t)$ is much smaller than its other dimensions: $2 \mathrm{~L}$-in the direction of the $\mathrm{x}$-axis and 2B-in the direction of the $\mathrm{y}$-axis.

The flanged area represents a plastic layer of constant thickness $h=h(t)$ and occupies an area bounded by the external contours:

$$
\mathrm{G} 1: f_{1}(x, y)=0 \text {. }
$$

For a deformable metal, the condition of complete plasticity is assumed $[1,3]$, according to which the tangent stress in the layer is small in comparison with normal

*Corresponding author: evgeny.sosenushkin@yandex.ru 
stresses and it can be ignored, and two normal stresses in the cross- section (vertical) in the initial approximation can be considered equal:

$$
\sigma_{x} \approx \sigma_{y}=-q ; \tau_{x y} \approx 0 .
$$

The third normal stress, constant in the thickness of the layer, is equal to the contact pressure of the layer on the tool:

$$
-\sigma_{z}=p
$$

and is determined from the plasticity condition $[1,6]$ :

$$
\sigma_{s}=p+q \text {. }
$$

To calculate the energy-power parameters of the process under study, it is proposed to use the theory of flow averaged over the thickness of the layer in a relatively thin plastic layer as described by A. A. Ilyushin [1]. We set a boundary value problem with respect to the contact pressure $p=p(x, y)$ and two components of the flow velocity $u=u(x, y)$ and $v=v(x, y)[1,3,6]$, which includes two quasi-static equilibrium equations and the incompressibility condition:

$$
\begin{aligned}
& \frac{\partial p}{\partial x}=-\frac{2 \tau_{s}}{h} \frac{u}{\sqrt{u^{2}+v^{2}}}, \\
& \frac{\partial p}{\partial y}=-\frac{2 \tau_{s}}{h} \frac{v}{\sqrt{u^{2}+v^{2}}}, \\
& \frac{\partial u}{\partial x}+\frac{\partial v}{\partial y}-\frac{d \lambda}{d t}=0,
\end{aligned}
$$

where $\lambda(t)=\ln \frac{h_{0}}{h(t)}$ is the degree of deformation according to A. A. Ilyushin (similarto the measure of accumulated deformation in a particle).

\section{Equations and mathematics}

In the forging under consideration, we select an element of symmetry $S_{i}=\left\{|(x, y)| a_{i 0} \leq x \leq a_{i 2} ; b_{i 0} \leq y \leq b_{i 1}\right\}$ with an external border (G1) in the form of walls into which the metal flows. In this case, the lines $x=a_{i 0}$ and $y=b_{i 0}$ serve as symmetry lines of the flow area (G2) and coincide with the axes of the selected coordinate system (Fig.1). In this case, the boundary conditions take the following form:

$$
\begin{aligned}
& G 1: f_{1}(x, y)=0, p=2 \tau_{s} \\
& G 2: v_{n}=0
\end{aligned}
$$

In this case, the external contour, where the metal flows freely, coincides with the level line (constant pressure). On the other hand, it is known $[1,3,6]$ that the current lines are 
straight, orthogonal to the level lines. Consequently, the quasi-static equation of equilibrium $\frac{\partial p}{\partial \xi}=\frac{2 \tau_{s}}{h}$ along the known flow line $\xi=\xi(x, y)$, is easy to integrate:

$$
p(\xi)=\frac{2 \tau_{s}}{h} \xi+2 \tau_{s}
$$

Thus, the contact pressure field in the considered flow stage is determined, which means that we can find the required force for the extrusion process. The solution of the problem of flow of a plastic layer of constant thickness $h=h(t)$ in the region bounded by the contour G1 is considered below. The selected area $S_{i}$ is further divided into subdomains $S_{i n}$ for convenience (see Fig. 1).

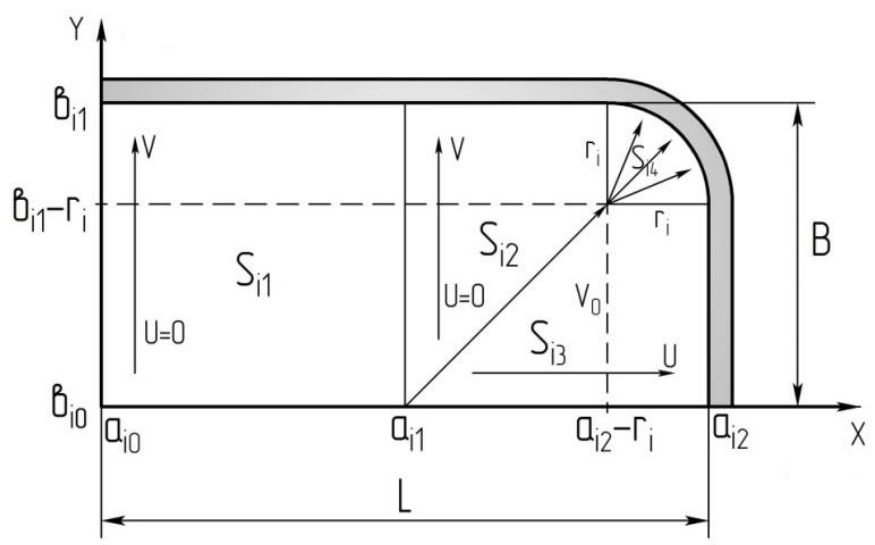

Fig. 1. Design scheme

The equations of the boundary value problem (5) - (7) for the specified subdomain $S_{i 1}=\left\{(x, y) \mid \begin{array}{l}a_{i 0} \leq x \leq a_{i 1} \\ b_{i 0} \leq y \leq b_{i 1}\end{array}\right\}$ are written:

$$
\begin{aligned}
& \frac{\partial p}{\partial x}=0 \\
& \frac{\partial p}{\partial y}=-\frac{2 \tau_{s}}{h_{i}} \\
& \frac{\partial v}{\partial y}=-\frac{1}{h_{i}} \frac{d h_{i}}{d t}
\end{aligned}
$$

The boundary conditions: $y=b_{i 0}=0: v=0 ; y=b_{i 1}: p=2 \tau_{s}$.

The pressure in the area is found by integrating the equation (12):

$$
p_{i 1}=2 \tau_{s}-\frac{2 \tau_{s}}{h_{i}}\left(y-b_{i 1}\right) .
$$

The component of the flow velocity after integrating the incompressibility equation (13) takes the form: 


$$
v_{i 1}=-\frac{1}{h_{i}} \frac{d h_{i}}{d t} y
$$

Stamping force in the area $S_{i 1}$ :

$$
P_{S_{i 1}}=\iint_{S_{i 1}} p_{i 1} d x d y=\left(a_{i 1}-a_{i 0}\right)\left[2 \tau_{s}\left(b_{i 1}-b_{i 0}\right)-\frac{\tau_{s}}{h_{i}}\left(b_{i 1}-b_{i 0}\right)^{2}\right]
$$

Let's consider the plastic area $S_{i 2}: S_{i 2}=\left\{\begin{array}{ll}(x, y) & \begin{array}{c}a_{i 1} \leq x \leq a_{i 2}-r_{i}, \\ x-\left(a_{i 0}-b_{i 0}\right) \leq y \leq b_{i 1}\end{array}\end{array}\right\}$

The equations of the boundary value problem are given in the form of the system (11) (13). Boundary conditions in the plastic area $S_{i 2}: y=x-\left(a_{i 0}-b_{i 0}\right): v=0 ; \quad y=b_{i 1}$ : $p=2 \tau_{s}$.

By analogy with the area $S_{i 1}$, the pressure is determined from equation (14):

$$
p_{i 2}=2 \tau_{s}-\frac{2 \tau_{s}}{h_{i}}\left(y-b_{i 1}\right)
$$

The flow rate is found by integrating the incompressibility equation (13):

$$
v_{i 2}=-\frac{1}{h_{i}} \frac{d h_{i}}{d t}\left[y-x+\left(a_{i 0}-b_{i 0}\right)\right] .
$$

Let's go to the definition of the stamping force in the area $S_{i 2}$ :

$$
\begin{aligned}
& P_{S_{i 2}}=\iint_{S_{i 2}} p_{i 2} d x d y=-\tau_{s}\left\{\left[\left(a_{i 2}-r_{i}-b_{i 1}\right)-\left(a_{i 0}-b_{i 0}\right)\right]^{2}-\left[\left(a_{i 1}-b_{i 1}\right)-\left(a_{i 0}-b_{i 0}\right)\right]^{2}\right\}+ \\
& +\frac{\tau_{s}}{3 h_{i}}\left\{\left[\left(a_{i 2}-r_{i}-b_{i 1}\right)-\left(a_{i 0}-b_{i 0}\right)\right]^{3}-\left[\left(a_{i 1}-b_{i 1}\right)-\left(a_{i 0}-b_{i 0}\right)\right]^{3}\right\}
\end{aligned}
$$

The next area of plastic flow $S_{i 3}: S_{i 3}=\left\{(x, y) \mid \begin{array}{c}y-\left(a_{i 0}-b_{i 0}\right) \leq x \leq a_{i 2} \\ b_{i 0} \leq y \leq b_{i 1}-r_{i}\end{array}\right\}$ form:

The equations of the boundary value problem (5) - (7) in the considered area have the

$$
\begin{aligned}
& \frac{\partial p}{\partial x}=-\frac{2 \tau_{s}}{h_{i}} \\
& \frac{\partial p}{\partial y}=0 \\
& \frac{\partial u}{\partial x}=-\frac{1}{h_{i}} \frac{d h_{i}}{d t} .
\end{aligned}
$$

The boundary conditions in the area $S_{i 3}: x=y-\left(a_{i 0}-b_{i 0}\right): u=0 ; x=a_{i 2}: p=2 \tau_{s}$.

The pressure in the area $S_{i 3}$ is found by integrating the equation (20): 


$$
p_{i 3}=2 \tau_{s}-\frac{2 \tau_{s}}{h_{i}}\left(x-a_{i 2}\right)
$$

The flow velocity is determined by integrating the incompressibility equation (22):

$$
u=-\frac{1}{h_{i}} \frac{d h_{i}}{d t}\left(x-a_{i 2}\right) .
$$

The stamping force in the area $S_{i 3}$ :

$$
\begin{aligned}
& P_{S_{i 3}}=\iint_{S_{i 3}} p_{i 3} d x d y=-\tau_{s}\left\{\left[\left(b_{i 2}-r_{i}-a_{i 1}\right)-\left(a_{i 2}-b_{i 0}\right)\right]^{2}-\left(a_{i 2}-a_{i 0}\right)^{2}\right\}+ \\
& +\frac{\tau_{s}}{3 h_{i}}\left\{\left[\left(b_{i 1}-r_{i}-a_{i 2}\right)-\left(a_{i 0}-b_{i 0}\right)\right]^{3}-\left(a_{i 0}-a_{i 2}\right)^{3}\right\}
\end{aligned}
$$

Let's consider the following plastic area $S_{i 4}$ :

$$
S_{i 4}=\left\{(x, y) \mid \begin{array}{c}
a_{i}-r_{i} \leq x \leq a_{i 2}, \\
b_{i 1}-r_{i} \leq y \leq\left(b_{i 1}-r_{i}\right)+\sqrt{r_{i}^{2}-\left[x-\left[a_{i}-r_{i}\right]\right]^{2}}
\end{array}\right\}
$$

For convenience, when performing calculations, we will proceed to the cylindrical coordinate system $[3,6]$, in which the limits of changing the geometric parameters are set:

$$
0 \leq \rho \leq r_{i} ; \quad 0 \leq \varphi \leq \frac{\pi}{2} .
$$

The boundary conditions in the area $S_{i 4}$ :

$$
\rho=r_{i}: p_{i 4}=2 \tau_{s} .
$$

The current lines form a bundle of straight lines centered at a point with coordinates $\left(a_{i 2}-r_{i} ; b_{i 1}-r_{i}\right)$.

The equations of the boundary value problem in the domain $S_{i 4}$ in cylindrical coordinates take the form:

$$
\begin{aligned}
& \frac{\partial p}{\partial \rho}=-\frac{2 \tau_{s}}{h_{i}} \\
& \frac{\partial p}{\partial \varphi}=0 \\
& \frac{\partial u}{\partial \rho}=-\frac{u}{\rho}-\frac{1}{h_{i}} \frac{d h_{i}}{d t}
\end{aligned}
$$

The contact pressure is determined from the integration of equation (28):

$$
p_{i 4}=2 \tau_{s}-\frac{2 \tau_{s}}{h_{i}}\left(\rho-r_{i}\right)
$$

The deformation force for the considered plastic area $S_{i 4}$ in the cylindrical coordinate system takes the form: 


$$
P_{S_{i 4}}=\iint_{S_{i 4}} p_{i 4} \rho d \rho d \varphi=\frac{\pi}{2}\left(\tau_{s} r_{i}^{2}+\frac{1}{3} \frac{r_{i}^{3}}{h_{i}}\right)
$$

\section{Results}

The paper presents an approach to calculating the power and kinematic parameters of the process using the example of box forging technology. A characteristic flow area is identified, for which the boundary value problem of plastic flow is formulated, based on the flow theory of A. A. Ilyushin. To solve the problem, the entire region $S_{i, j}(x, y)$ of the plastic flow is divided into the sum of subdomains with a characteristic metal flow, which allowed us to obtain a complete analytical solution of the problem. Using the presented results, it is possible to construct areas of more complex configuration and calculate the results using original numerical methods and software packages based on the obtained result.

\section{Conclusion}

The presented mathematical model allows us to determine the nature of the change and estimate the pressure values at the contact of the deformable metal with the tool, as well as the values and intensity of changes in the force and deformation parameters of the technological process of extrusion of box forgings.

\section{References}

1. A.A.Ilyushin,Plasticity (Fizmatlit, Moscow, 2004)

2. L.Prandtl, ZAMM, J3(6)(1923)

3. I.A.Kiyko. Theory of plastic flow(Mosc. Un-t, 1978)

4. D.V.Georgiyevsky, Mech of SolidsJ1. (2006)

5. V.A.Kadymov, Math Methods in the Appl SciJ38,16(2015)

6. V.A.Kadymov Math modeling of contact problems of plastic flow(Palmarium Ac. Publishing, 2016)

7. E.N. Sosenushkin, V.A.Kadymov, E.A. Yanovskaya, T.V. Gureeva,Extrusion mechanics of an aluminium alloy forging with longitudinal ribs, Tsvetnye Metally. 3(915),69-75 (2019) 